\title{
Por un sentido de la crítica literaria
}

\section{For a sense of literary criticism}

\author{
Francisco Estévez Regidor \\ Universidad de Málaga, España \\ festevez@uma.es
}

Resumen: El presente artículo medita sobre algunos particulares de la evolución que a lo largo de lo de los últimos decenios ha experimentado la grafomanía y, con ella, la crítica literaria al ser considerada como "fuente secundaria". Además, se pone en relación esta idea de crítica como literatura vicaria con el proceso de saturación interpretativa contemporánea. Movimiento éste que, sin duda, junto a otros han conseguido desplazar la posición del crítico hacia zonas de indeterminación, complicando el sentido final de la propia crítica literaria y su ubicación errática, ya desplazada del eje central de reflexión sobre la literatura en la era digital.

Palabras clave: crítica literaria, teoría, era digital, evolución, saturación.

Abstract: The present paper reflects on some particulars of the evolution the graphomania has undergone over the last decades and, with it, literary criticism as it is considered a "secondary source". Furthermore, this idea of criticism as vicarious literature is related to the contemporary critical saturation process. Movements that, without a doubt, have also displaced the critic's position towards zones of indeterminacy, complicating the final meaning of literary criticism itself and its erratic location, already displaced from the central axis of reflection on literature in the digital age. 
Keywords: Literary criticism, Literary theory, Digital age, Evolution, Saturation.

Recibido: 17 de marzo de 2020

Aceptado: 10 de mayo de 2020 https://dx.doi.org/10.15174/rv.vi26.537

W $\begin{aligned} & \text { ichel de Montaigne ya consideró en sus Ensayos como "se } \\ & \text { invierte más tiempo en interpretar las interpretaciones que }\end{aligned}$ en interpretar las cosas, y hay más libros sobre libros que sobre cualquier otro asunto" (III, Cap. XIII). Superada la primera mitad del siglo xx, Alfonso Reyes, con su habitual sagacidad, atisbó en sus primeros estadios contemporáneos el problema que ya se arrastraba de antaño y que enunció al escribir, precisamente y no por casualidad, El deslinde: Prolegómenos a la teoría literaria (1963), donde apreciaba que en cualquier tema literario la inmensa cantidad de fuentes secundarias -ya sean estos monografías, artículos, ensayos, etc.- dificultaba sobremanera abarcar con una sola vida cualquier objeto de estudio:

se ha escrito tanto sobre todas las cosas que la sola consideración de la montaña acumulada en cada área de saber produce escalofríos y desmayos, y a menudo oculta los documentos primeros de nuestro estudio, los objetos mismos y las dos o tres interpretaciones fundamentales que bastan para tomar el contacto (Reyes, 1963: 18).

Apenas nueve años después, y cual pelota de nieve en cuesta, la materia libresca había crecido de manera exponencial hasta un punto crítico que instigó a Gabriel Zaid a reflexionar con demole- 
dor título Los demasiados libros (1972) ${ }^{1}$, y no poco lamento pues allí cercenaba o al menos mutilaba la literatura crítica, el ensayo, las fuentes "secundarias", la obra compuesta con miras al mercado $y$, en definitiva, cualquier forma libresca de la grafomanía univer$\mathrm{sal}^{2}$ como posibilidad de comprensión: "Tratando de entender la cuestión central, de buscar soluciones, de refutar convencionalismos, mi propia grafomanía me ha puesto en contradicción: añadir uno más a los demasiados libros" (1996: 10). En efecto, parece que la época dorada de la Literatura tocaba un techo tras transitar con la gran novela europea el siglo XIx, arribar a la Modernidad del xx y, pongamos, hasta mediados del siglo. Así las cosas, quizás pudiéramos considerar a Borges como uno de los últimos capaz de afirmar aquello de "que otros se jacten de las páginas que han escrito; a mí me enorgullecen las que he leído". De tal manera que, durante el último cuarto de siglo $\mathrm{xx}$, el exceso abrumador de originales parece desmontar aquella idea clásica por la cual un libro debería tener miles de lectores. George Steiner ha sido quizá uno de los primeros gavieros en plantear el futuro del mundo editorial en el capítulo "Después del libro, ¿qué?", incluido en Sobre la dificultad y otros ensayos (1978): "Reformulada por Horacio y por Ovidio, la promesa de que el tiempo no puede carcomer las grandes palabras hasta volverlas polvo, de que perdurarán más que el bronce y el mármol en que están grabadas, es la contraseńa de la literatura occidental" (285) y semejante apuesta por la inmortali-

\footnotetext{
${ }^{1}$ El texto se ha reeditado numerosas veces en distintas editoriales Océano, Anagrama, Diana, El Colegio Nacional, Lumen, Debolsillo, con ańadidos y correcciones. Aquí es citado por la edición de Anagrama de 1996.

${ }^{2}$ El monteregiano salvaba de la pira a "los libros que nos acompañan: los viejos libros de ser releídos (los clásicos) y los contemporáneos inspirados en esa tradición" (10) queda patente el alineamiento estricto con Harold Bloom frente a posturas de relativismo cultural.
} 
dad solo puede sustanciarse "si el lenguaje mismo se sostiene" ${ }^{3} \mathrm{He}$ aquí en síntesis la concepción que tendría mejor desarrollo, con pespuntes azorados en la escritura del inglés en Presencias reales, de 1989, camino que terminó en Pasión intacta (1997): "Indagar sobre el estatus del libro y sobre el enigma de la revelación en el lenguaje significa toparse una y otra vez con el judaísmo y su trágico destino". En esta postura vemos cierta transustanciación, nunca mejor dicho por aquel matiz teológico y en el cual es fácil constatar la inteligente denuncia que realizaba Edward Said: "los críticos no solo crean los valores mediante los cuales se juzga y se comprende el arte, sino que encarnan en la escritura aquellos procesos y condiciones reales del presente mediante los cuales el arte y la escritura transmiten significado" (2013: 77). Pues a la postre, la idea que subyace entre líneas es una idea de texto puro, de primera lectura, ese primer motor aristotélico reflejado en el libro clásico como si éste fuera una obra virginal, adánica, como ha sabido objetar Mario Lavagetto (2005). En Literatura no tenemos una obra original, todas son texturas, interpretaciones, comentarios o glosas de otras anteriores. Es por ello que el intento de prescindir de obras secundarias o de la crítica literaria redunda a todas luces en una concepción teológica de la Literatura. Steiner es claro:

Estoy imaginando una república contraplatónica en la que los críticos y reseñadores han sido prohibidos; una república para escritores y lectores [...] en cambio estaría la crítica de arte, las reseñas periodísticas de pintores, escultores o arquitectos [...] la prohibición principal haría referencia a las reseñas, las críticas y las interpretaciones discursivas [...] En resumen, estoy construyendo una sociedad, una política de lo primario (1992: 16-17).

${ }^{3}$ Sin embargo, la postura de Steiner contiene más perfiles, al admitir que "Todo arte, música o literatura serios constituyen un acto crítico” (1992: 23). 
Véase aquí la degradante concepción que Steiner plantea de la crítica, quien a la postre debe matizarla a duras penas cuando "el problema que surge del hecho de que toda explicación es, en alguna medida, valorativa y crítica”. Y sin embargo, corrido el tiempo desde entonces, el follaje no ha dejado de crecer por entre la mesa de novedades de las librerías sin posibilidad alguna por parte del crítico o del lector de desbrozar una senda, menos aún de distinguir la flor extraña única.

Frente a la sobreabundancia viene repitiéndose una queja sorda que ha tenido como mejor cauce la reflexión de Antonio Valdecantos en Misión del ágrafo. Allí se advierte que es tarea consustancial al ágrafo reducir la producción misma de discurso; el silencio entendido como ejercicio de generosidad. Aconseja el filósofo meditar sobre el arraigado tabú por el cual la inmensa mayoría de lo escrito y publicado yace "sin haber sido leído nunca por nadie ni llegará a serlo jamás” (Valdecantos, 2016: 64). Este tabú resulta de vital importancia para la crítica literaria, tenida como literatura secundaria, vicaria y prescindible al ser producida gracias a otro texto anterior, pero ¿no resulta esta acaso la definición propia de literatura o debemos cancelar por entero toda la teoría de la intertextualidad? ¿Es posible acaso sacudir de un manotazo la perspicaz teoría de Mijail Bajtin y con él los posteriores desarrollos que hicieran en su traducción y explicación al mundo francés y europeo tanto Tzvetan Todorov como Julia Kristeva?

Pero eliminado ese grueso nudo, si como alumbra Valdecantos la expectativa lógica de cualquier texto resulta el estar abocado a la sorda desatención, cuestión que parece tener a todas luces peor perspectiva al ser colgado en la red, destinado a "la soledad más onanista" de lo cual se deduce que la idea de publicación es una construcción ficticia fundada en una especie de "Panóptico textual” (Valdecantos: 2016: 57-71). Así vista por estas consideraciones previas, la lectura resulta siempre más un acto en potencia que 
una realidad de facto. Si bien es cierto que su simple posibilidad de acaecer mantiene por entero toda la ficción para engrasar la pesada maquinaria editorial. Desvertebrado de tal manera el homo Gutenberg y llegados los arrestos digitales no sabemos cuál es el horizonte de actividad y sentido de la crítica literaria.

Alguna pista pudiera darnos el hecho de que los textos apenas interpretados líneas arriba, con sus peculiaridades intrínsecas y matices, remiten al pasaje célebre de Platón cuando en el Fedro expone en boca de Sócrates el mito egipcio de la escritura encarnado por el dios Theut que se lo muestra a su padre Thamus. De la medicina para la memoria al veneno para la memoria: cara y cruz de la escritura. Aunque Platón subordine la escritura a la oralidad queda siempre la propia incongruencia de Platón ante la necesidad de poner por escrito el propio Fedro no siguiendo el ejemplo de su amado Sócrates. Pero, en esencia quedan la escritura y, su revés, la lectura, como una tecnología que permite almacenar "memoria", es decir, información, y en pasos siguientes reelaborarla, trasmitirla o en su defecto archivarla y sepultarla, ocultarla. En aquel pasaje, Platón se fija en buena cantidad de detalles pero pasa por alto la importancia del lector en la decodificación del mensaje como vértice último del proceso artístico. Si ponemos mientes en la teoría de la recepción otorgar peaje a la radical sentencia de Pessoa "El único prefacio a una obra es el cerebro de quien la lee” invalidaría en buena parte la oportunidad de muchas reflexiones. Por suerte, como sabemos, en terreno artístico y en el intelecto del ser humano, el colorido de los matices desbanca la simplificación en blanco y negro de los extremos binarios. Sin duda, es correcta la dirección hacia donde apunta Pessoa. Todo lector proyecta su experiencia, su cultura, sus conocimientos, pero sobre todo, su sensibilidad, al texto literario conformando un sentido único a la lectura emprendida. ¿Acaso una obra de creación no surge como respuesta a otra anterior, como comentario crítico, prolongación, debate, 
de una predecesora? También es cierto, y en sentido contrario a Pessoa, que la crítica orienta, propone distintos senderos, descubre pistas de lectura. Hoy parece que la frase "aquel que posee talento es naturalmente inclinado a la crítica, porque ve más cosas que los otros y las ve mejor" del Barón de Montesquieu resulta en franca decadencia dentro de la república de las letras. Resulta como si la influencia del crítico en las letras viniera trufada de insospechados vaivenes. En España, por ejemplo, durante el siglo XIX se produjo un crecimiento ostensible de la profesión hermenéutica a pesar de que los Pirineos actuaron de sordina frente al restallido de la frase de Kant, "Atrévete a pensar", que muy atenuada arribó a la piel de toro. Nombres como los de Leopoldo Alas Clarín, Juan Valera, Manuel de la Revilla o el polígrafo Marcelino Menéndez Pelayo constituyeron una pléyade crítica que otorgó convicción y autoridad al crítico tras las influencias desmedidas del romanticismo. Se desmontaba aquella creencia por la cual el autor, inconsciente de su quehacer, atendía solo el susurro falsario de las musas para escribir. Desde entonces la crítica descansó sobre los pilares de la cultura y el sentimiento estético educado del crítico se alejó de esa suerte de intuición ajena a los principios generales del arte que la caracterizó en su origen. Bastaría destacar, entre otras posibles, las figuras señeras de Northrop Frye y Roland Barthes para evidenciar el extraordinario desarrollo de la crítica literaria durante la segunda mitad del siglo $x x$.

Sin embargo, desde inicios del 2000 la progresiva erosión del término "crítica", la pérdida de su poder valorativo y la limitación de su influencia pueden observarse con frecuencia sin necesidad de lupa alguna. El cambio de paradigma tecnológico, a la postre cultural, espoleado por una presunta democratización de la información sustentada por internet y otros factores difíciles de sintetizar aquí someten al análisis crítico de la cultura y, en particular, de la literatura a una corrosiva devaluación. Los cambios que ha traído 
consigo la sociedad digital a todo el campo literario, Bourdieu mediante, obvian por completo la reflexión sobre el papel del crítico literario en la sociedad, el alcance de su oficio y la validez de sus postulados. Semejante análisis conduce de manera inexorable a una pregunta que nos concierne a todos. La formuló antaño Ortega y Gasset donde expuso su doctrina sobre la crítica literaria: “¿Qué es leer?” entonada en su célebre primer libro Meditaciones del Quijote (1914). ${ }^{4}$ Reflexionaba del siguiente modo sobre la crítica:

La crítica no es biografía ni se justifica como labor independiente, si no se propone completar la obra. Esto quiere decir, por lo pronto, que el crítico ha de introducir en su trabajo todos aquellos utensilios sentimentales e ideológicos pertrechado con los cuales puede el lector medio recibir la impresión más intensa y clara de la obra que sea posible. Procede orientar la crítica en un sentido afirmativo y dirigirla, más que a corregir al autor, a dotar al lector de un órgano visual más perfecto. La obra se completa completando su lectura (1914: 52).

En definitiva, y siguiendo las ideas de Ortega y Gasset, la crítica vendría a crear una atmósfera de lectura conveniente al lector, dicha atmósfera estaría íntimamente conectada a la concretización propuesta por Wolfgang Iser por la cual el lector funda un significado concreto de entre los posibles en su lectura del texto y también conectada a la interpretación situacional histórica propuesta por Gadamer, a fin de cuentas un diálogo entre pasado y presente. Cierto es, cada época se realiza esta pregunta basilar según sus premisas epocales y su horizonte de entendimiento o ¿acaso fue

4 "Del mismo modo que hay un ver que es un mirar, hay un leer que es un intelligere o leer lo de dentro, un leer pensativo. Solo ante este se presenta el sentido profundo del Quijote" (89). 
casual que solo superada la II Guerra Mundial Jean Paul Sartre pudiera teñir de compromiso la pregunta en su ensayo ¿Qué es la literatura? de1948?

Por otro lado, en la actualidad, de entre los peligros que dinamitan el quehacer crítico actual parece sustantiva la sospecha de dilentantismo. A través de los nuevos medios, las redes, los fanzines electrónicos, revistas digitales, blogs y otras páginas personales se procede sin cesar a "Criticar al crítico", por parafrasear el título de un estimulante ensayo de T. S. Eliot. Allí se desacredita a la crítica oficial para en el fondo instaurar una nueva crítica donde el difamador lógicamente tenga la posición de poder. Nada nuevo en nuestros pagos donde la crítica es siempre acerba, en especial consigo misma. Sin embargo, entendida en su mejor esencia, la crítica y toda escritura de género análogo aspira a encantar, a dar una visión limpia del texto referido, una lectura luminosa por el grado intenso de apreciación estética. Pregunta legítima es cuestionar si puede hacer algo más o si es exigible que vaya más allá del cumplimiento de sus deberes connaturales. Y, en efecto, así es. Va más allá al enseñar y alguna vez, fíjese en el diverso matiz de la palabra y en la timidez del uso, alguna vez, aspira a educar. Esa elemental tarea a pie de obra literaria, siendo vicaria, se eleva como insustituible y necesaria. Por inextricable que parezca el sino de la crítica y por más que al crítico se le achaque afanar el templo de la literatura. Ciertamente, en ocasiones, parece que los artistas desearan ser los únicos encargados de administrar la religión pertinente. El discurso literario atańe a muchos otros convidados y de forma preeminente al lector, polo último de significación del hecho literario, el cual viene atendido por los mimos del crítico. "No hay hechos, solo interpretaciones", advertía Nietzsche en su Genealogía de la moral.

Es una declaración de principios, en primera instancia, repetir si es necesario hasta la saciedad frente al relativismo omnímodo 
una idea vertebradora del célebre ensayo Los limites de la interpretación de Umberto Eco (1990). Todas las interpretaciones no son iguales, ni guardan el mismo respeto frente al hecho artístico al que aluden. Parece de Pero Grullo y quizá por ello hemos transigido tal obviedad: si todas las interpretaciones tuvieran el mismo valor, ninguna sería valiosa. Vale decir: la diferencia es el nivel, construye el grado, queramos o no un escalafón. De otra manera, el valor es también en la medida de su rareza, por así decirlo.

Si la docencia literaria de antaño tiene hoy día una muy restrictiva cabida en los planes de estudio universitarios; si la enseñanza literaria resulta hoy incapaz de justificar la centralidad de su utilidad; si las humanidades, en suma, se asoman al vértigo de la indeterminación y el presente de las cosas resulta así para el profesor de literatura, qué tiempos corren para el crítico literario. Parece que la sombra de Heidegger en su Carta sobre el humanismo, de 1947, planea sobre nosotros. Aclarados estos burdos preliminares, avancemos una verdad de nuestros tiempos: el discurso mediático permea cada vez con mayor profusión el discurso académico. Podemos discutir el grado de cumplimiento de este axioma, pero no su existencia. Si algo caracteriza al enigmático concepto de posmodernismo es la mezcolanza de discursos. Y si tal ocurre en la Academia no menos o con mayor insistencia fuera de sus límites donde los discursos son más proclives a la banalización.

La noción de crítica, al ser histórica, resulta inestable y, por ende, más allá de la disciplina que entendemos por tal; pero si prescindamos por un momento del conjunto de saberes acumulados y los debates que en el pasado y presente la acotan, cabe la posibilidad de entenderla como el conjunto de actos atenientes a valorar el hecho literario. La ostensible erosión experimentada por la crítica literaria ha fomentado el surgimiento de nuevas formas críticas más acordes con los tiempos y las obras del día. Sin embargo, parte considerable de la crítica literaria actual es sospechosa 
de dilentantismo, cuando no de simple intrusismo, donde la única centralidad es la disparidad absoluta de criterios valorativos. Si bien el solipsismo parece arraigar fuerte en la escritura de muchos jóvenes. Nuestro pensamiento camina contra el uso de los tiempos (Cervantes dixit) ya que comprender una obra alude de forma inmediata a dos direcciones: el sentido y la alteridad. No en vano Gadamer tanteaba siempre en sus libros el arte de la comprensión, frente al sinsentido, el consentimiento, la aceptación del otro, para referirse a la hermenéutica, comprender siquiera de forma tentativa, trazar puentes hacia la alteridad del texto que permitan a potenciales lectores acomodar sus pasos a cierta guía. De modo más claro, o más clásico, el crítico anhela mediar entre lector y autor para que éstos simpaticen, claro está, simpatía en su sentido etimológico, griego, en el sentido de comprensión de lo otro, de ir más allá. Éste debiera ser el hilo rojo que guiará los esfuerzos hermenéuticos.

El mundo literario actual parece vivir una continua querella de viejos y jóvenes adaptada a los tiempos que corren en tecnófobos y geeks, versión moderna de aquel Apocalípticos e integrados de Eco (1964). Como toda disputa falsea por reducción la realidad (pensemos por ejemplo la oquedad de aquella disputa entre un supuesto noventayochismo tosco y un modernismo extremadamente sensible) y obvia una parte considerable del espectro creativo que sigue aún los canales tradicionales. Sin embargo, es cierto que el tiempo de la literatura cambia de constante y que la cultura tal y como la conocemos está siempre en permanente crisis. Los cambios en el mercado literario, en la producción y la difusión de las obras, no modifican la esencia literaria ni siquiera su persistencia según parece. Más bien, los cambios vienen registrados en el "campo literario" y ese dichoso fenómeno magnificado por el mundo de internet: casi todo el mundo escribe. Ello tiene una derivación inmediata y es que la novedad literaria -el valor de la literatura 
ante la saturación de producto- se convierte en algo mucho más complicado de discernir y el despiste general entre lectores resulta a veces mayúsculo. En tales circunstancias, se evidencia el papel intermediador del crítico y es deseable revitalizar su figura. Tal vez hubiera que recordar cómo fue en otras épocas ese augur cultural, augur en clara referencia de nuevo al mundo clásico, a ese oficiante que en la antigua Roma practicaba la adivinación por el canto, el vuelo o la manera de comer de las aves entre otros signos. En nuestro caso, augur porque desentraña no aves, sino libros y ausculta en sus páginas el latir de la época. Así de serio y arriesgado resulta el oficio del crítico cuando se atreve a leer el espíritu de los tiempos. Tal atrevimiento es en la mayoría de los casos una osadía pues acecha el riesgo de deslumbramientos o de error, a cada cual peor.

De otro costado España es un país sin lectores que de forma curiosa continúa con un nutrido y valioso número de escritores. $\mathrm{Al}$ mismo tiempo, como durante al menos todo el siglo xx, la internet no ha hecho más que radicalizar tal situación, se publica y se escribe más de lo que infinitamente se puede leer. Todo el mundo ansía escribir y, de un modo u otro, por algún canal consigue hacerlo, siquiera en blogs. Ahora bien, cuánto se lee, mucho menos de lo que podríamos intuir, me temo. No podemos engatusarnos con cantos de sirena. Cualquier estadística pone los pies en el suelo y no resulta difícil comprobar como en Espańa tenemos una media aproximada de unos 25.000 lectores reales.

Ante esta situación, la crítica literaria, queramos o no, es la judicatura de toda esa desbordante literatura (y la patronal es sin duda el mundo editorial). Ese lector cualificado que es el crítico debe mediar entre la obra de arte y el lector común para ayudarle a que la entienda mejor y la disfrute. Esta simple definición pondría en entredicho gran parte de los mejores esfuerzos académicos que hoy día se aplican a interpretar y "explicar" la literatura contemporánea, pues vienen envueltos en jergas ininteligibles para aquel que 
no se haya dado al fútil y arduo trabajo de aprenderla previamente, como ya se ha advertido en otras ocasiones. El esoterismo en terrenos críticos es la más de las veces síntoma de incomprensión. Los griegos no enseńaban a sus iguales nada que un niño no pudiese entender. Mediar entre los productos literarios y el lector de a pie es la función hermenéutica. La crítica debe juzgar, condenar o alabar, pero sobre todo, analizar, no proporcionar informaciones huecas que nadie solicita. La creación crítica, pues, en estas orillas del amor al texto no consiste en inventar, sino en descubrir.

De otro modo, todos pueden tener una opinión sobre un libro, pero no tantos pueden emitir un juicio sobre el mismo. El juicio se argumenta, la opinión se posee. La diferencia entre ambos es la distancia que media entre la impresión de un hecho estético y su reflexión. La senda siempre es trazada por alguien en primer lugar. El crítico en nuestro caso, para que los demás puedan hacer camino, a su manera. Según Pessoa, cada lector pone frente al libro lo que tiene: el teórico, ideas; enseńanzas, el maestro, ideales el sońador, etc. Sin embargo, el crítico debe tejer una sábana que envuelva todas esas posibles decodificaciones y ofrecer ánimo y calor al lector para entrar en el texto reseñado cuando éste resulte de valía o realizar las salvedades y precauciones necesarias ante libros que no alcancen ciertos mínimos. En suma, orientar al público. Para ello se precisa una independencia de espíritu que aborde la lectura del libro a través de su propia experiencia y no por la opinión ya configurada. Se necesita valentía moral para ir contra la corriente general.

Parecen lejanos los días en que un escritor reputado como Torrente Ballester y poco conocido en su faceta como crítico y teórico pensaba en los primeros días de 1990 que "nadie discute la legitimidad de la crítica” (59). Hoy día, el péndulo ha oscilado en

${ }^{5}$ Entre otros libros de teoría cabe destacar el póstumo Teoría de la novela. 
sentido contrario y poca es la legitimidad residual que tiene aquel arte vicario y menos aún los abanderados que abogan por la necesidad de la crítica cuando hoy en realidad resulta más apremiante por una nefasta huida hacia delante editorial que implica publicar mucho más de lo aceptable. La verdad es que no solo el público necesita la crítica, sino también el autor. El juicio ajeno es siempre solicitado incluso nuestro mayor genio literario ya atisbo tal necesidad encarnada en el mismísimo D. Quijote cuando pregunta a su amigo Sancho:

¿Qué es lo que dicen de mí por ese lugar? ¿En qué opinión me tiene el vulgo, en qué los hidalgos, y en qué los caballeros? ¿qué dicen de mi valentía, qué de mis hazañas y qué de mi cortesía?” y más adelante inquiere al Bachiller ¡Qué hazañas mías son las que más se ponderan en esa historia? -En eso-respondió el Bachillerhay diferentes opiniones, como hay diferentes gustos [...] Una de las tachas que ponen a la tal historia -dijo el Bachiller- es que su autor puso en ella una novela intitulada El curioso impertinente; no por mala ni por mal razonada, sino por no ser de aquel lugar, ni tiene que ver con la historia de su merced don Quijote [...] -dijo don Quijote- [...] y no sé yo qué le movió al autor a valerse de novelas y cuentos ajenos, haciendo tanto que escribir en los míos (parte II, capítulo II).

La actividad cultural que conforma la crítica literaria es siempre hija de su tiempo y quizás en este periodo de desguace de las $\mathrm{Hu}$ manidades que atravesamos hayamos de volver al concepto desgranado por Baudelaire en la crónica de los salones de arte en París, aquel por el cual toda crítica de arte ha de ser personal, apasionada y política. Françoise Perus afirmó que al analizar la literatura la cuestión era no tanto "ordenar las obras en función de su mayor o menor grado de adecuación con las sucesivas 'esencias' que va 
elaborando la crítica idealista seducida por sus propios espejismos, sino en restituir a los hechos literarios reales su historicidad concreta"

De una forma más genérica relacionamos de forma sencilla el aspecto de la crítica como una actitud intelectual necesaria y de primer orden no solo en ambiente cultural, sino en cualquier rincón del ágora pública para vitalizar una democracia entendida en su sentido más exigente y no en los actuales que parecen ser decorado cartón piedra. Si toda obra de arte tiende a recoger la realidad general de su tiempo y tiende a transformar el orden del mundo, la crítica que reciba esa obra debe valorar éticamente tal perspectiva estética. La función de una crítica de altura, aún aquella de carácter ocasional como las hojas volanderas de un suplemento periodístico debe atisbar tales funciones, ofrecer pistas de lectura y debe apuntarse en equilibrio por un profundo sentido de la continuidad de la cultura en la que se integra la obra analizada.

La misión de la crítica fue para Pedro Salinas, autor de La voz a mí debida como dijera con chascarrillo pero no falto de razón un soberbio Juan Ramón, revivir o recrear esa experiencia en su más hondo y pleno significado. No tanto pediremos a la crítica inmediata, aunque recalcaremos que criticar implica juzgar, lo cual equivale a situar la novela o el poemario e interpretar el texto. Esclarecer siempre. Lo decía Machado en su famosa definición de poesía, es "palabra esencial en el tiempo". Lo requería Guillermo de Torre para la crítica, lo que corresponde es ver las obras en el plano de su interpretación con el tiempo y, sin embargo, toda visión valedera deberá tomar como punto de referencia nuestra posición concreta (la del crítico) en un espacio y un momento dados so riesgo de falsear la correcta perspectiva. Según el acertado ensayista, el profetizar es algo que solo puede tener en nuestros días una traducción menos délfica, más humana para el crítico: equivale sencillamente a juzgar, a situar y valorar, combinando los datos de 
un presente y las intuiciones de un futuro que las circunstancias tornan ya igualmente hipotético y azaroso.

La tendencia secular fue una crítica a rebufo de la altura de su tiempo, con excelentes excepciones como la de Clarín. El péndulo de la historia parece situarse con la misma inclinación. La concepción hermenéutica actual prefiere la glosa o paráfrasis antes del análisis concienzudo. De tal modo, el crítico sí merece aquel conocido dicterio de Valle Inclán tan delatador "hembras y los eunucos del Arte". Internet ha abierto varias brechas tanto en el lado creativo como en el terreno crítico, pero los problemas siguen siendo los mismos. Internet no debe dirigirnos hacia el sinsentido, la crítica cuando es creativa nos acerca al consentimiento y a la comprensión de la alteridad del texto. En definitiva, trasladar las sutilezas del arte literario a la crítica solicita otras sutilezas. Pero siempre quedará la obra y si la crítica incentiva en algo es para buscar entre anaqueles la novela, el poemario, aquel drama, en las estanterías, en las librerías o las bibliotecas públicas o privadas allí encontraremos lo que, las más de las ocasiones, el crítico quizá no supo transmitir.

\section{Bibliografía}

Barthes, Roland, 1984, "La muerte del autor", en El susurro del lenguaje. Más allá de la palabra y la escritura, Paidós, Barcelona. Eco, Umberto, 1990, Los limites de la interpretación, Lumen, Barcelona.

, 1968, Apocalípticos e integrados, Lumen, Barcelona.

Eliot, Thomas Stearns, 2011, La aventura sin fin, Lumen, Barcelona.

Frye Northrop, 1977, Anatomía de la critica, Monte Ávila, Caracas. 
Gadamer, Hans Georg, 1977-2002, Verdad y Método, I y II [19601982].

Heidegger, Martin, 1959, Carta sobre el humanismo, Taurus, Madrid.

Nietzsche, Friedrich, 1971, Genealogía de la moral, Alianza, Madrid.

Ortega y Gasset, José, 1914, Meditaciones del Quijote: meditación preliminar, meditación primera, Imp. Clásica española, Madrid.

Perus, François, 1982, Historia y critica literaria (el realismo social y la crisis de la dominación oligárquica, Casa de las Américas, La Habana.

Pessoa, Fernando, 2013, Escritos sobre genio y locura, Acantilado, Barcelona.

Reyes, Alfonso, 1963, El deslinde, en Obras Completas, t. XV, Fondo de Cultura Económica, México.

Said, Edward, 2004, El mundo, el texto y el crítico, Debate, Buenos Aires.

Sartre, Jean Paul, 1957, ¿Qué es la literatura?, Losada, Buenos Aires.

S/f., 1992, Presencias reales, Editorial Destino, Barcelona.

Steiner, George, 2002, Sobre la dificultad y otros ensayos, Fondo de Cultura Económica, México.

Todorov, Tzvetan, 2005, Crítica de la crítica, Paidós, Barcelona.

Torrente Ballester, Gonzalo, 1990, "La crítica como necesidad", $A B C, 14$ de julio. ,2017, Teoría de la novela, Deliberar, Madrid.

Valdecantos, Antonio, 2016, Misión del ágrafo, José Manuel Cuesta Abad (pról.), La uÑa RoTa, Segovia. 
Valle Inclán, Ramón María del, 1904, Prólogo de Sonata de primavera: Memorias del Marqués de Bradomin, Madrid.

Zaid, Gabriel, 1972, Los demasiados libros, Carlos Lohlé, Buenos Aires. 